\title{
Striatum Inhibition: Clues for Cocaine Addiction Therapy
}

\author{
Hayet Boudjani, Stéphane Potvin, Céline Chayer, Simon Charbonneau, Sylvain Lanthier
}

doi:10.1017/cjn.2014.13

Can J Neurol Sci. 2014; 41: 664-665

The human brain's reward system is regulated mainly by three interacting dopaminergic pathways: the mesolimbic and mesocortical pathways projecting from the ventral tegmental area to the nucleus accumbens (ventral striatum) and the prefrontal cortex and the mesostriatal pathway projecting from the substantia nigra to the dorsal striatum. ${ }^{1}$ Dopamine concentration in the reward circuitry rises when one experiences rewarding or gratifying feelings and decreases with perceptions of unpleasantness. Blockade of presynaptic dopamine transporters by cocaine prevents dopamine reuptake, allowing prolonged dopaminergic stimulation. Recent studies suggest that the mesostriatal pathway, especially involving the dorsolateral striatum (DLS), plays a more important role than previously thought in cocaine addiction. ${ }^{1} \mathrm{We}$ report a clinical case highlighting the impact of a dorsal striatal lesion on cessation of craving for cocaine in a human, suggesting that deep brain stimulation (DBS) might represent a possible future treatment for cocaine addiction.

\section{Case Report}

A 45-year-old, right-handed male consulted for acute right-sided hemiparesis with dysarthria, which started improving 1 hour after onset. His medical history was unremarkable, except for hepatitis $\mathrm{C}$ and traits of antisocial personality. He had been incarcerated several times and reported having regular contacts with prostitutes. He started smoking cigarettes and cannabis and using other illicit drugs at age 9 . Since age 24 , the patient used phencyclidine regularly and intravenous or inhaled cocaine (generally $3.5 \mathrm{~g} / \mathrm{d}$, up to $7 \mathrm{~g} / \mathrm{d}$ ). $\mathrm{He}$ reported headaches with a continuous and intense daily urge to consume cocaine since starting behavioral therapy for drug abstinence a month before presentation.

Physical examination revealed skin tattoos, poor oral hygiene with a dental abscess, no fever, normal heart sounds, no systemic signs of endocarditis, and a residual neurological deficit limited to a mild proportional right-sided weakness. Brain MRI showed an acute nonlacunar infarct of the left putamen with slight extension to rostral pallidum, caudate body, and corona radiata, and a dilated Virchow-Robin space in the right basal ganglia (Figure 1A). The nucleus accumbens was spared. Computed tomography angiography revealed dissection of the subpetrosal segment of the left internal carotid artery with pseudoaneurysm. Transthoracic and transesophageal echocardiography documented mitral vegetations. Serial blood cultures were negative. Results of Holter monitoring, erythrocyte sedimentation rate, C-reactive protein, and other blood tests for vasculitis and prothrombotic states were unremarkable. We diagnosed acute ischemic stroke of the left basal ganglia secondary to nontraumatic internal carotid artery dissection. A cardioembolic etiology from aseptic mitral valve vegetations remaining from previous endocarditis was considered less likely. Aspirin and clopidogrel were started.

In the subsequent days, his right-sided weakness resolved completely, revealing micrographia (Figure 1B). He denied any cognitive or mood change except for a reduction in impulsiveness. The patient claimed to have no craving for cocaine or any other stimulants since the onset of his stroke. He continued to smoke cigarettes and cannabis. He remained abstinent for cocaine except twice in 39 months after the stroke, driven by social convenience among his friends. He experienced the expected stimulant effects of cocaine but without any subsequent cravings. Fifteen weeks after his stroke, the patient retrospectively scored $9 / 10$ on the Drug Abuse Screening Test for the year preceding the stroke, indicating severe drug-related problems. He scored $0 / 10$ for the period after the stroke, indicating no residual problems of drug use. Five months after the stroke, he tested positive for cannabis but negative for cocaine and other street drugs in an "unannounced" toxicology screening, which was undertaken at his second of seven follow-up visits.

\section{DiscuSSION}

We report that an acute lesion involving mainly the left dorsal striatum resulted in sudden and persistent interruption of a longlasting seeking behavior for psychostimulant drugs. This unique human case complements functional neuroimaging and animal studies highlighting the central role of the dopaminergic mesostriatal reward circuitry in conditioned reinforcement and cocaine addiction. Functional neuroimaging studies in human have shown that cocaine cues increase dopamine in the striatum. ${ }^{2}$ There is some evidence that there are both distinguishable and intermingled roles of the dorsal and ventral striatum in cocaine addiction. ${ }^{2,3}$ It appears that dopamine levels in the dorsal, but not in the ventral, striatum predicts the severity of craving in cocaine addiction in humans. ${ }^{2}$ Given the equivocal efficacy of dopaminergic agents for treating human addiction, nondopaminergic mechanisms may be involved in the increasingly acknowledged physiological role of the DLS in compulsive drug-seeking, such as neuroplastic or glutamatergic changes. ${ }^{4}$ Consistent with what is

From the Department of Neurosciences (HB, CC, SL) and Department of Psychiatry (SP), Faculty of Medicine, Université de Montréal; Department of Medicine (HB, SL) and Psychology (SC), CR-CHUM; Fernand-Seguin Research Centre, Louis-H. Lafontaine Hospital Centre (SP), and Department of Medicine, Hôpital Maisonneuve-Rosemont (CC), Montreal, Quebec, Canada.

Received January 8, 2014. Final Revisions Submitted April 11, 2014. Correspondence to: Sylvain Lanthier, CHUM - Hôpital Notre-Dame, 1560 East Sherbrooke Street, Suite 1166, Montreal, Quebec, H2L 4M1, Canada.

E-mail: sylanthier@gmail.com 


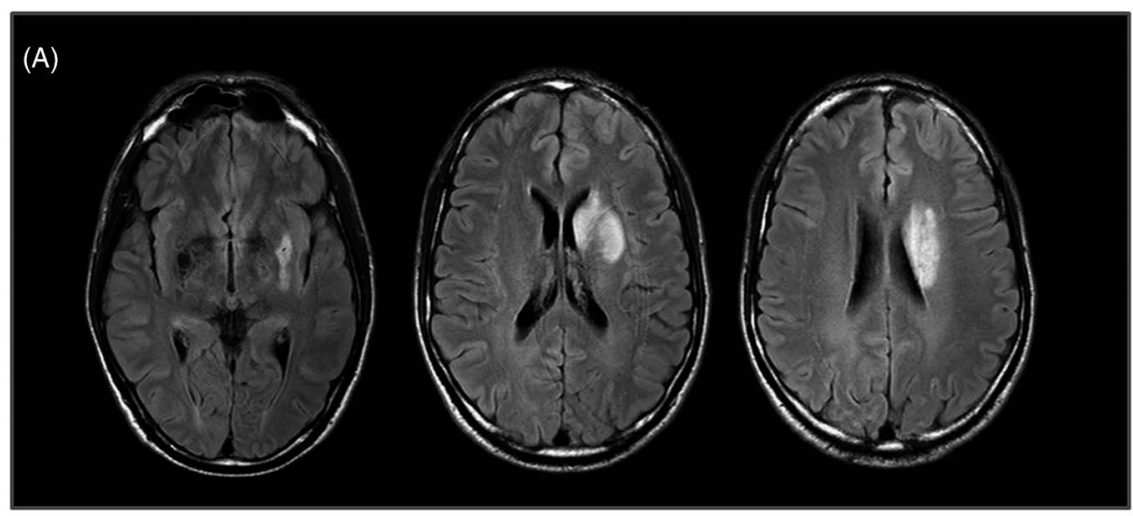

(B)

$$
\begin{aligned}
& 24 \text { days before stroke: } \\
& \text { Ce si est } \mathcal{L} \text { 'histoise de ma vie }
\end{aligned}
$$

\section{4 months following stroke:}

$$
\text { ae si est blustion de ma sie }
$$

Figure 1: (A) Fluid-attenuated inversion recovery sequences in a brain MRI scan showing an acute infarct of the left putamen with extension to rostral pallidum, caudate body, and corona radiata, and a dilated Virchow-Robin space in the right basal ganglia. (B) Comparison of the patient's writing before and after the stroke showing an acquired micrographia (Sentence written in French, with spelling errors: This is the story of my life).

seen in our patient, experimental lesion studies in animals have shown that DLS lesions attenuate cocaine-seeking behaviors and inhibit cocaine reinstatement. ${ }^{3}$ Furthermore, results from intrastriatal disconnection studies in rats confirm that the inactivation of the DLS, but not its surrounding structures, impairs cocaine-seeking behaviors. ${ }^{3}$ Research with animal models and preliminary observations in human suggest that the nucleus accumbens and the subthalamic nucleus are potential targets for treatment of drug addiction by DBS. ${ }^{5}$ In light of our case, the DLS represents another potential anatomic target. That routine toxic screening was not obtained at each follow-up visit is a potential limitation to our study.

Another interesting manifestation in this patient is the presence of micrographia as an isolated, persistent extrapyramidal sign. Few cases of isolated micrographia have been reported in association with infarct of the contralateral putamen and adjacent area. Micrographia is related to dopamine depletion in Parkinson's disease. Micrographia may further reflect a disrupted dopamine circuitry in our case of cocaine addiction reversal.

This unique case report substantiates the central role of the dorsal striatum in cocaine addiction in humans. We believe that treatments designed to target specific neural structures, such as DBS, represent a promising therapeutic option for cocaine dependence.

\section{ACKNOWLEDGEMENTS}

The authors thank the CR-CHUM Editorial Service for reviewing this manuscript.

\section{REFERENCES}

1. Wise RA. Roles for nigrostriatal—not just mesocorticolimbicdopamine in reward and addiction. Trends Neurosci. 2009; 32(10):517-24.

2. Volkow ND, Wang GJ, Telang F, et al. Cocaine cues and dopamine in dorsal striatum: mechanism of craving in cocaine addiction. J Neurosci. 2006;26(27):6583-8.

3. Belin D, Everitt BJ. Cocaine seeking habits depend upon dopaminedependent serial connectivity linking the ventral with the dorsal striatum. Neuron. 2008;57(3):432-41.

4. Grueter BA, Rothwell PE, Malenka RC. Integrating synaptic plasticity and striatal circuit function in addiction. Curr Opin Neurobiol. 2012;22(3):545-51.

5. Luigies J, van den Brink W, Feenstra M, et al. Deep brain stimulation in addiction: a review of potential brain targets. Mol Psychiatry. 2012;17(6):572-83. 